\title{
The Antibiotic Prescribing Pathway for Presumed Urinary Tract Infections in Nursing Home Residents
}

\author{
Christine E. Kistler, MD, MASc, ${ }^{\star}$ Sheryl Zimmerman, PhD, ${ }^{\dagger}$ Kezia Scales, PhD, ${ }^{*}$ Kimberly Ward, BA,,$^{\S}$ \\ David Weber, MD, "L David Reed, PhD, ${ }^{\S}$ Mallory McClester, MD, * and Philip D. Sloane, MD, MPH ${ }^{\S}$ *
}

OBJECTIVES: Due to the high rates of inappropriate antibiotic prescribing for presumed urinary tract infections (UTIs) in nursing home (NH) residents, we sought to examine the antibiotic prescribing pathway and the extent to which it agrees with the Loeb criteria; findings can suggest strategies for antibiotic stewardship.

METHODS: Chart review of 260 randomly-selected cases from $247 \mathrm{NH}$ residents treated with an antibiotic for a presumed UTI in $31 \mathrm{NHs}$ in North Carolina. We examined the prescribing pathway from presenting illness, to the prescribing event, illness work-up and subsequent clinical events including emergency department use, hospitalization, and death. Analyses described the decision-making processes and outcomes and compared decisions made with Loeb criteria for initiation of antibiotics.

RESULTS: Of 260 cases, $60 \%$ had documented signs/ symptoms of the presenting illness and $15 \%$ met the Loeb criteria. Acute mental status change was the most commonly documented sign/symptom $(24 \%)$. NH providers $(81 \%)$ were the most common prescribers and ciprofloxacin $(32 \%)$ was the most commonly prescribed antibiotic. Fourteen percent of presumed UTI cases included a white blood cell count, $71 \%$ included a urinalysis, and $72 \%$ had a urine culture. Seventy-five percent of cultures grew at least one organism with $\geq 100,000$ colony-forming units/ milliliter and $12 \%$ grew multi-drug resistant organisms;

From the *Department of Family Medicine, School of Medicine; ${ }^{\dagger}$ School of Social Work, Cecil G. Sheps Center for Health Services Research, University of North Carolina, Chapel Hill; ${ }^{\text {SS}}$ chool of Nursing, Duke University, Durham; ${ }^{\S}$ Cecil G. Sheps Center for Health Services Research; and "Department of Medicine, University of North Carolina, Chapel Hill, North Carolina.

This research was carried out by the Program on Aging, Disability and Long-Term Care of the Cecil G. Sheps Center for Health Services Research at the University of North Carolina at Chapel Hill. Portions of these findings were presented at the 2015 Gerontological Society of America conference and the 2016 American Geriatrics Society annual conference.

Address correspondence to Christine E. Kistler, Department of Family Medicine, School of Medicine, Cecil G. Sheps Center for Health Services Research, University of North Carolina, Chapel Hill, NC, 27599. E-mail: christine_kistler@med.unc.edu

DOI: $10.1111 /$ jgs. 14857
$28 \%$ of antibiotics were prescribed for more than 7 days, and $7 \%$ of cases had a subsequent death, emergency department visit, or hospitalization within 7 days.

DISCUSSION: Non-specific signs/symptoms appeared to influence prescribing more often than urinary tract-specific signs/symptoms. Prescribers rarely stopped antibiotics, and a minority prescribed for overly long periods. Providers may need additional support to guide the decision-making process to reduce antibiotic overuse and antibiotic resistance. J Am Geriatr Soc 65:1719-1725, 2017.

Key words: older adults; antibiotic stewardship; decision-making

Suspected urinary tract infections (UTIs) in adults aged $\bigcirc 65$ years and older account for over 8 million office visits and 1 million emergency department visits, with estimated annual treatment costs exceeding $\$ 2.3$ billion. ${ }^{1,2}$ Few clinical problems pose more decision-making difficulties than the management of suspected $\mathrm{UTIs}^{3-5}$ due to the absence of a diagnostic gold standard exist and high rates of asymptomatic bacteriuria (ASB). ${ }^{6,7}$ Also, significant disagreement exists in clinical practice as to what constitutes a UTI. ${ }^{8}$

Among nursing home $(\mathrm{NH})$ residents, clinical decision-making for suspected UTIs poses additional challenges for a variety of reasons. For one, ASB rates are particularly high in NHs; as many as half of all positive urine cultures should be considered false positives for the presence of a UTI. $^{9}$ Additionally, due to high rates of dementia among $\mathrm{NH}$ residents, nurses must often infer the presence of symptoms based on nonverbal responses. ${ }^{10}$ Further, NH providers, such as physicians, physicians' assistants, and nurse practitioners, often make decisions with extremely limited information, not knowing an individual resident's medical history ${ }^{11,12}$ or not examining a resident at the time of illness presentation. ${ }^{13}$ Although evidence shows that clinical prescribing guidelines for UTIs such as the Loeb criteria can reduce overprescribing in $\mathrm{NHs},{ }^{14}$ broader 
evidence suggests that $\mathrm{NH}$ nurses and providers often deviate from guidelines when making antibiotic prescribing decisions. ${ }^{15-18}$ Instead of relying on evidence-based guidelines, they also consider non-specific signs and symptoms (e.g., lack of appetite) and non-symptom information such as a patient or family request for an antibiotic, ${ }^{15,16,18}$ which can foster overprescribing. ${ }^{19}$

Looking at the prescribing pathway (the resident's presenting illness episode, the prescribing event, the work-up, and subsequent clinical events) may shed light on the importance of each component of the pathway and provide targets for future interventions. Previous studies have been small or focused on only one part of the process. A prospective study of $100 \mathrm{NH}$ residents evaluated the association between three common diagnostic guidelines for UTI and positive urine cultures. ${ }^{20}$ The positive predictive value of these guidelines was around $60 \%$. Another study conducted in $4 \mathrm{NHs}$ over a 6 -month period found that almost half of all antibiotic prescriptions for suspected UTI occurred in residents with no documented symptoms and that clinical decision-making often rested solely on urine culture results. ${ }^{6}$ Given the small number of NHs in these studies, further work is critically needed to understand this pathway.

As part of a larger study of antibiotic stewardship, we collected baseline data to understand the prescribing pathway for presumed UTIs. We examined documented clinical symptoms (particularly the Loeb criteria), prescriber characteristics, antibiotic prescribed, rates of antibiotic cessation after a negative urine culture, and subsequent clinical events. We aimed to determine the frequency of prescriptions that met Loeb criteria, and the rate of discontinuation of antibiotics in the face of negative urine cultures.

\section{METHODS}

\section{Study Design}

Part of a dissemination effort to improve infection management and antibiotic stewardship, we enrolled $31 \mathrm{com}$ munity-based NHs in North Carolina. At baseline a team of geriatricians and research staff visited each $\mathrm{NH}$ between November 2014 and March 2015 to conduct medical record audits. In each $\mathrm{NH}$, we randomly selected ten "new" cases of treated UTIs from the infection log for the preceding month. A new case was defined as an antibiotic prescription for a UTI without a prior prescription for a UTI in the past 21 days, assuming that antibiotics clustered closer together were a part of the same illness episode and not discrete UTIs. In NHs having fewer than ten cases in the month, all available cases were audited, yielding a total of 260 cases. The University of North Carolina Institutional Review Board approved the study. The funding source had no role in the study.

\section{Population}

To be eligible for study participation, cases had to be recorded on each NH's infection control $\log$ and resulted in a systemic antibiotic for a presumed UTI. Residents were eligible regardless of whether they were in short-term rehabilitation or long-term stay beds.

\section{Measures}

We developed an abstraction form to record elements in the antibiotic prescribing pathway. The form included information about the presenting illness episode (i.e., vital and physical signs and symptoms), the prescribing event (i.e., the type and administrative route of the antibiotic, where it was prescribed and by whom), the illness workup (i.e., laboratories, urinalysis and urine culture performed, and results), the antibiotic treatment course (i.e., when started and changed or stopped, and duration), and subsequent clinical events such as emergency department use, hospitalization, and death (in the 7 days after the antibiotic was initiated). Descriptive information about the $\mathrm{NHs}$ and residents also were obtained.

We created three variables from the existing data: (1) whether the presenting illness met the minimum set of prescribing criteria developed by Loeb et al. ${ }^{21}$ (2) whether at least one of the Loeb criteria was present; and (3) whether the cultured organism met criteria for a multi-drug resistant organism per the Centers for Disease Control criteria. ${ }^{22}$ The Loeb minimum criteria for prescribing are consensus-guidelines and differ depending on whether or not a resident has an indwelling-catheter. ${ }^{14,21}$ In residents with indwelling urinary catheters, a patient is considered to have a UTI and a prescription is appropriate if the resident has either a fever (temperature $>100^{\circ} \mathrm{F}$ ) or new onset of costovertebral angle tenderness, symptoms of rigors, or new symptoms of delirium. If there is not a catheter, a patient is considered to have a UTI and a prescription is appropriate if the resident has either pain or difficulty with urination alone, or fever and at least 1 of the following: new or increased urinary urgency, new or increased urinary frequency, new or increased suprapubic pain, new costovertebral angle tenderness, frank hematuria, or new or worsened urinary incontinence. We conducted an additional analysis using the Loeb criteria but considering fever to be $\geq 99.0^{\circ} \mathrm{F}$ instead of $>100^{\circ} \mathrm{F}$, based on recent evidence. $^{23} \mathrm{~A}$ positive urine culture was considered $\geq 10,000$ colony-forming units/milliliter $(\mathrm{CFU} / \mathrm{mL})$ in those with a documented catheterized specimen and $\geq 100,000 \mathrm{CFU} / \mathrm{mL}$ for all other specimens. In terms of determining multi-drug resistance, if results of the urine culture and sensitivities were not present, the results were presumed to be negative.

\section{Data Analysis}

Descriptive statistics were used to examine the data, including frequencies, percentages, means, and standard deviations (SD). When the classification of symptom and signs was unclear, two authors (CK and PS) reviewed the data and assigned them to existing categories if they independently agreed on their reassignment. CK summarized the 17 cases of emergency department use, hospitalization, and death. All quantitative analyses were conducted using SPSS (Armonk, NY: IBM Corporation), version 23.

\section{RESULTS}

Of the $31 \mathrm{NHs}$, most were for-profit $(77 \%)$ with a mean size of 109 licensed beds (Table 1). During the 1-month 
Table 1. Nursing Home and Resident Characteristics Characteristics

n (\%) or Mean (SD)

$\begin{array}{lc}\text { Nursing home characteristics }(\mathrm{n}=31) & 15(48 \%) \\ \text { Nursing home chain } & 16(52 \%) \\ \text { Long-term care medical practice }^{\mathrm{a}} & 24(77 \%) \\ \text { For-profit } & 5(16 \%) \\ \text { Has a dementia unit } & 109 \pm 44 \\ \text { Number of licensed beds per site, } & \\ \text { mean } \pm \text { SD } & 2 \pm 1 \\ \text { Staff turnovers in prior } 5 \text { years (number per site), mean } \pm \text { SD } \\ \text { Administrator } & 3 \pm 2 \\ \text { Director of Nursing } & 2 \pm 2 \\ \text { Infection control nurse } & 2 \pm 1 \\ \text { Medical Director } & 29 \pm 14 \\ \text { Licensed nurses (RN/LPN) } & \\ \text { Resident characteristics }(n=247)^{\mathrm{b}} & 13(36-110) \\ \text { Age, mean } \pm \text { SD (range) } & 184(75 \%) \\ \text { Gender, female } & 15(6 \%) \\ \text { On hospice } & \\ \text { Routine catheter use } & \\ \text { Indwelling urinary catheter } & 37(15 \%) \\ \text { Routine intermittent catheterization } & 5(2 \%) \\ \text { Suprapubic catheter } & 13(5 \%) \\ \text { None of the above } & 179(73 \%) \\ \text { Unknown } & 13(5 \%)\end{array}$

$\mathrm{SD}=$ Standard Deviation; $\mathrm{RN}=$ Registered Nurse $;$ LPN = Licensed Practi cal Nurse.

${ }^{\text {a }}$ Long-term care medical practice is a group of medical providers including physicians, physician assistants, and nurse practitioners who provide care only to $\mathrm{NH}$ residents.

${ }^{5}$ Thirteen residents had repeated treated UTIs, resulting in a total of 260 cases of treated UTIs.

${ }^{\mathrm{c}}$ Excludes ileal conduit.

study period, we reviewed $247 \mathrm{NH}$ residents' charts who received 260 antibiotic prescriptions for a presumed UTI, with $13(5 \%)$ residents receiving two prescriptions. Most of the $\mathrm{NH}$ residents who received an antibiotic were female $(75 \%)$ and without routine catheter use $(73 \%)$, with a mean age of 81 (range 36-110).

\section{Presenting Illness}

Overall, $60 \%$ of cases had at least one sign or symptom documented in the 48 hours prior to when the prescription was written. Of the 156 cases with documented signs or symptoms, $106(68 \%)$ met at least one Loeb criterion (41\% of all cases; see Table 2). In total, $15 \%$ met all Loeb criteria, and when we used a less stringent criterion for fever (temperature $\geq 99.0^{\circ} \mathrm{F}$ ), $17 \%$ met criteria. The most common urinary tract-specific sign or symptom was dysuria $(9 \%)$. Overall, acute mental status change was the most commonly documented sign or symptom $(24 \%)$.

\section{Prescribing Event}

$\mathrm{NH}$ residents most commonly received oral antibiotics $(87 \%)$, with ciprofloxacin being the most common prescription $(32 \%)$, followed by trimethoprim-sulfamethoxazole $(14 \%)$ and nitrofurantoin $(11 \%)$ (see Table 3). Most antibiotics were prescribed by $\mathrm{NH}$ providers $(81 \%)$. A minority were prescribed during a hospital inpatient stay
Table 2. Characteristics of the Illness Presentation Documented in the Nursing Home Record $(\mathrm{n}=260)$

\section{Characteristic}

n (\%) or Mean (SD)

\begin{tabular}{|c|c|}
\hline \multicolumn{2}{|l|}{ Vital signs and symptoms } \\
\hline \multicolumn{2}{|l|}{ Lowest systolic blood pressure } \\
\hline$<100$ & $11(4 \%)$ \\
\hline$>140$ & $21(8 \%)$ \\
\hline No blood pressure recorded & $93(36 \%)$ \\
\hline Highest temperature recorded, mean \pm SD & $98.5 \pm 1.1$ \\
\hline Solitary temperature $>100.0^{\circ} \mathrm{F}\left(37.8^{\circ} \mathrm{C}\right)$ & $18(7 \%)$ \\
\hline Two recorded temperatures $>99.0^{\circ} \mathrm{F}\left(37.2^{\circ} \mathrm{C}\right)$ & $17(7 \%)$ \\
\hline \multicolumn{2}{|l|}{ Physical signs and symptoms } \\
\hline Dysuria & $24(9 \%)$ \\
\hline Urgency & $4(2 \%)$ \\
\hline New/increased frequency & $7(3 \%)$ \\
\hline CVA (costovertebral angle) tenderness & $9(4 \%)$ \\
\hline Suprapubic tenderness & $6(2 \%)$ \\
\hline $\begin{array}{l}\text { Pain, swelling, or tenderness of testes, } \\
\text { epididymis, or prostate }^{\mathrm{a}}\end{array}$ & $0(0 \%)$ \\
\hline New/increase incontinence & $5(2 \%)$ \\
\hline Hematuria & $8(3 \%)$ \\
\hline Pus around catheter & $2(1 \%)$ \\
\hline Acute mental status change & $61(24 \%)$ \\
\hline Acute functional decline & $24(9 \%)$ \\
\hline Change in urine color & $25(10 \%)$ \\
\hline Change in odor & $20(8 \%)$ \\
\hline Other & $71(27 \%)$ \\
\hline \multicolumn{2}{|l|}{ Minimum criteria for antibiotic initiation $\left(\right.$ Loeb $\left.^{b}\right)$} \\
\hline Meet Loeb ${ }^{b}$ criteria for UTI & $43(17 \%)$ \\
\hline Meet $\geq 1$ Loeb criteria & $106(41 \%)$ \\
\hline
\end{tabular}

$\mathrm{SD}=$ Standard Deviation; UTI $=$ Urinary Tract Infection.

${ }^{\text {a}}$ Sample included 67 males.

${ }^{\mathrm{b}}$ To meet Loeb criteria, a resident must have acute dysuria alone or fever (>99.0F) plus at least one of the following: new or worsening urgency, new or worsening frequency, suprapubic pain, gross hematuria, costovertebral angle tenderness, and new or increased urinary incontinence.

$(10 \%)$, emergency department visit $(6 \%)$, or visit to a medical office $(2 \%)$. Of the 194 prescriptions for whom the prescriber's profession was noted, physicians prescribed $52 \%$, nurse practitioners prescribed $38 \%$, and physician's assistants prescribed $10 \%$.

\section{Illness Work-Up}

As part of the illness work-up, white blood cell (WBC) counts, urinalyses, and urine cultures were performed for some residents. Fourteen percent of cases had a WBC count performed within the 3 days prior to the antibiotic (Table 4). Of the $71 \%$ who had a urinalysis performed, $10 \%$ were negative and $29 \%$ were both nitrite and leukocyte esterase positive. Similarly, $72 \%$ of cases had a urine culture performed, of which $3 \%$ were unknown, $3 \%$ resulted no bacteria, and $4 \%$ were polymicrobial. Ninety percent of cultures resulted some type and quantity of organism; $78 \%$ were considered positive cultures based on our definition. Forty three of the 70 cases missing a urine culture $(59 \%)$ were prescribed outside of the NH. Cases diagnosed in the $\mathrm{NH}$ were more likely to have an available urine culture $(P<.001)$. Escherichia coli $(E$. coli) resulted most often (34\%), followed by Klebsiella $(14 \%)$ and Proteus $(12 \%)$ as shown in the Figure 1. Multi-drug resistant 
Table 3. Characteristics of the Prescribing Event Documented in the Nursing Home Record $(n=260)$

\section{Characteristic}

\begin{tabular}{lc} 
Antibiotic prescribed & \\
Ciprofloxacin & $82(32 \%)$ \\
Trimethoprim-sulfamethoxazole & $36(14 \%)$ \\
Nitrofurantoin & $28(11 \%)$ \\
Penicillin & $27(10 \%)$ \\
Third-generation cephalosporin & $26(10 \%)$ \\
Other fluoroquinolone & $20(8 \%)$ \\
Other & $19(7 \%)$ \\
\hline First-generation cephalosporin & $16(6 \%)$ \\
Second-generation cephalosporin & $5(2 \%)$ \\
Fourth-generation cephalosporin & $1(<1 \%)$ \\
Route of antibiotic & \\
Oral & $227(87 \%)$ \\
Parenteral (IV or IM) & $29(11 \%)$ \\
Other/missing & $4(2 \%)$ \\
Location of antibiotic prescription & \\
Nursing home & $211(81 \%)$ \\
Hospital & $27(10 \%)$ \\
Emergency department & $15(6 \%)$ \\
\hline Outside provider & $6(2 \%)$ \\
Antibiotic prescriber & $100(39 \%)$ \\
Physician & $74(29 \%)$ \\
Nurse practitioner & $20(8 \%)$ \\
Physician's assistant & $66(25 \%)$ \\
Unknown/missing &
\end{tabular}

IV = Intravenous; IM = Intramuscular.

organisms (MDROs) were found in $12 \%$ of positive cultures, and only $7 \%$ of organisms were pan-sensitive. Although not statistically significant, residents with indwelling catheters were more likely to be diagnosed with an MDRO than those without ( $8 \%$ vs. $4 \%, P=.43$ ).

\section{Antibiotic Treatment Course}

Antibiotics were prescribed for a mean duration of 7.5 days $(\mathrm{SD} \pm 2.7) ; 28 \%$ of prescriptions exceeded 7 days, $3 \%$ exceeded 10 days, and $9 \%$ were for 5 days or less. In total, $2 \%$ of prescriptions were discontinued, and $19 \%$ were changed during the illness course. On average, residents with indwelling catheters received antibiotics for 8.4 days $(95 \%$ CI $7.5,9.2)$ as compared to those without who received antibiotics for 7.2 days (95\% CI 6.9, 7.5) $(P=.008)$.

\section{Clinical Events}

Of the 247 cases, $7 \%$ had a subsequent clinical event. Two died during the course of treatment, eleven required hospitalization, and 4 required emergency department visits. Of the 11 hospitalized residents, $5(45 \%)$ had 1 or more of the signs or symptoms found in the Loeb criteria and three $(27 \%)$ met the Loeb criteria. One of the residents who died was on hospice.

\section{DISCUSSION}

In this study of $31 \mathrm{NHs,} \mathrm{examination} \mathrm{of} \mathrm{the} \mathrm{prescribing}$ pathway for presumed UTIs revealed a number of findings that may explain why adherence to inflectional control guidelines continues to be poor and how each contributes to inappropriate antibiotic prescribing and the development of MDROs. As a sign of potentially inappropriate prescribing, non-specific signs or symptoms, particularly acute mental status change, were more commonly found with antibiotic prescriptions than the expert-derived Loeb criteria for antibiotic initiation. Most antibiotics were prescribed by $\mathrm{NH}$ providers, and antibiotics were rarely discontinued before the course was completed. In addition, for a sizeable minority, the duration of antibiotics appeared overly long (i.e., $28 \%$ were prescribed for more than 7 days, which exceeds the recommended duration of 3-7 days. ${ }^{24,25}$ Among positive cultures, 92\% grew an organism resistant to at least one antibiotic.

Diagnostic errors along the pathway appear to play a significant role in inappropriate antimicrobial prescribing in $\mathrm{NH}$ residents, as also seen in hospitalized patients. ${ }^{26}$ One of the early components of the prescribing pathway, namely signs and symptoms of the presenting illness, may represent an important area for intervention. Despite the existence of evidence-based guidelines for more than a decade ${ }^{21}$ we continue to see that most presumed UTIs fail to meet minimum requirements for appropriate antibiotic initiation. ${ }^{10,18,27,28}$ Admittedly, one possible explanation for apparent inappropriate prescribing may be the lack of documentation in $\mathrm{NH}$ charts. Even when symptoms were documented, however, non-specific signs and symptoms such as confusion or agitation (which have shown little to no association with $\mathrm{UTIs}^{29}$ ) were documented most frequently. Clinical assessments in the $\mathrm{NH}$ are particularly difficult. Due to high rates of dementia, nurses often must coax symptom reports from residents. ${ }^{10}$ Nurses may note a patient's non-specific change in condition, which then sets off a cascade of information gathering and further decisions, including calling a physician, that result in an antibiotic prescription. $\mathrm{NH}$ providers often have limited familiarity with a $\mathrm{NH}$ resident, ${ }^{11,12}$ and may not examine a resident with a suspected UTI until days after an antibiotic has been initiated. In one study, only $29 \%$ of residents with unstable vital signs were examined by a provider onsite or prior to transfer to a hospital. ${ }^{13}$ Further, although $78 \%$ of providers awaited culture results prior to prescribing, we found limited evidence that residents were reassessed to determine if the clinical picture warranted a UTI diagnosis or if the culture results represented ASB. Given the non-specific nature of many signs or symptoms used to diagnose suspected UTIs, a period of watchful waiting with non-pharmacologic management, such as timed toileting and oral hydration, with a reassessment of the resident's status may provide a reasonable approach to reducing unnecessary prescribing.

Most of these prescriptions (81\%) were written by $\mathrm{NH}$ providers, whose knowledge of appropriate antibiotic prescribing is historically low. ${ }^{30}$ To address this issue, efforts such as clinical decision support systems (CDSS) or peer evaluations may improve prescribing quality and safety in NHs. A systematic review of CDSS in NHs to improve medication safety ${ }^{31}$ found that CDSS use for residents with renal insufficiency significantly increased appropriate medication orders by $20 \% .^{32}$ A recent RCT of 47 primary care practices testing a CDSS to support 
Table 4. Characteristics of the Illness Work-up and Antibiotic Treatment Course ${ }^{a}$

All Cases $(n=260)$ n (\%) or Mean (SD)

Met Any Loeb Sign/Symptoms $(n=106)$ n (\%) or Mean (SD)

Met Loeb Criteria $(n=43)$ $\mathrm{n}(\%)$ or Mean (SD)

\begin{tabular}{|c|c|c|c|}
\hline Laboratory tests performed & & & \\
\hline White blood cell count, mean \pm SD & $9,772 \pm 4,132$ & $9,985 \pm 4,425$ & $13,200 \pm 4,315$ \\
\hline Left shift present & $1(3 \%)$ & $1(4 \%)$ & $1(11 \%)$ \\
\hline Presence of bands not reported & $34(94 \%)$ & $25(93 \%)$ & $7(78 \%)$ \\
\hline Urinalysis performed & $184(71 \%)$ & $85(80 \%)$ & $33(77 \%)$ \\
\hline Nitrite, positive (moderate, large) & $72(39 \%)$ & $37(44 \%)$ & $16(48 \%)$ \\
\hline White blood cells, moderate or large & $103(56 \%)$ & $51(60 \%)$ & $22(67 \%)$ \\
\hline Red blood cells, moderate or large & $29(16 \%)$ & $14(16 \%)$ & $11(33 \%)$ \\
\hline Specific gravity, mean (SD) & $1.017(0.006)$ & $1.016(0.006)$ & $1.015(0.006)$ \\
\hline Urine culture performed & $186(72 \%)$ & $87(82 \%)$ & $37(86 \%)$ \\
\hline Negative for bacteria (no CFU/mL) & $6(3 \%)$ & $3(3 \%)$ & $0(0 \%)$ \\
\hline Polymicrobial organisms reported & $7(4 \%)$ & $6(7 \%)$ & $3(8 \%)$ \\
\hline Unknown (report not found) & $5(3 \%)$ & $4(5 \%)$ & $0(0 \%)$ \\
\hline Multi-drug resistant organism ${ }^{b}$ & $16(12 \%)$ & $6(10 \%)$ & $4(13 \%)$ \\
\hline Antibiotic course & $260(100 \%)$ & $106(100 \%)$ & $43(100 \%)$ \\
\hline $\begin{array}{l}\text { Antibiotic started prior to urine } \\
\text { culture results }\end{array}$ & $58(22 \%)$ & $42(40 \%)$ & $19(44 \%)$ \\
\hline Antibiotic changed & $48(19 \%)$ & $30(28 \%)$ & $16(37 \%)$ \\
\hline Antibiotic stopped & $4(2 \%)$ & $2(2 \%)$ & $0(0 \%)$ \\
\hline Antibiotic duration, mean days \pm SD & $7.5 \pm 2.7$ & $7.3 \pm 2.9$ & $7.3 \pm 3.9$ \\
\hline
\end{tabular}

${ }^{a}$ Bold values are percentages of the total in the column header. Roman values are percentages of the $\mathrm{n}$ in bold above it (except see footnote ${ }^{\mathrm{b}}$ ).

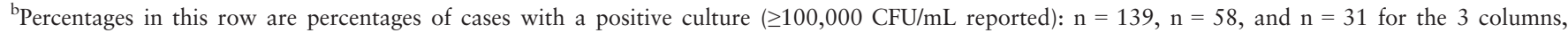
respectively.

${ }^{\mathrm{c}} \mathrm{Six}$ residents were reportedly catheterized for the specimen.

prescribing for upper respiratory infections also found antibiotics prescribing fell significantly compared to controls. ${ }^{33}$ Consequently, work is needed to see if targeted CDSS for the diagnosis of infections can be translated from the outpatient setting to the $\mathrm{NH} .^{34}$

Other steps in the pathway may benefit from potential interventions. In hospital settings, discontinuing reflexive urine cultures has reduced antibiotic prescribing. ${ }^{35} \mathrm{~A}$ similar intervention may be useful in NHs. Even when faced with negative cultures, providers apparently failed to reexamine their diagnosis and discontinue antibiotics-despite the fact that discontinuing antibiotics, when no infection is present, reduces antibiotic resistance. ${ }^{36}$ Outpatient treatment guidelines for UTIs in the United Kingdom explicitly state that antibiotics should be discontinued if a culture yields no bacterial count. ${ }^{28,37}$ Yet our providers discontinued antibiotics in only 4 of 6 cases with no bacterial growth. Standing orders for antibiotic cessation when urine cultures are negative may reduce antibiotic use. Additionally, interventions addressing catheter-associated UTIs (CAUTIs), such as a nursing-led program to discontinue indwelling catheters in hospitalized patients, could be useful in NHs. ${ }^{38}$ We found that residents with catheters more often had MDROs than those without. Given the push by federal agencies for antibiotic stewardship programs in $\mathrm{NHs},{ }^{39}$ systems to stop orders for unnecessary urine cultures, to reassess the clinical scenario in the face of culture results, or discontinue catheter use after a period of time might be indicated in NHs.

Not only do providers prescribe regardless of clinical presentation in some cases, but their choice of antibiotics and the duration of treatment are also sometimes inappropriate. Our study shows continued frequent prescribing of fluoroquinolones ( $40 \%$ of cases) and less-frequent prescribing of recommended first-line treatments ( $25 \%$ of cases). ${ }^{6}$ Antibiotic resistance continues to be high in NHs, and MDROs are more common. Almost half of E. coli found in significant cultures was resistant to ciprofloxacin or levofloxacin. This resistance pattern is worrisome given the need to safeguard levofloxacin for cases of bacterial pneumonia, as it is one of the few oral antibiotics recommended for health-care associated pneumonia. ${ }^{40}$ The Infectious Disease Society of America (IDSA) recommends that nitrofurantoin, trimethoprim-sulfamethoxazole, fosfomycin, or pivmecillinam be used before a fluoroquinolone (e.g., ciprofloxacin or levofloxacin) or betalactam. ${ }^{41}$ Fluoroquinolones are discouraged because of side effects ranging from neurologic toxicity to cardiac abnormalities, and cross-resistance between ciprofloxacin and levofloxacin is nearly universal. ${ }^{42}$ The Food and Drug 


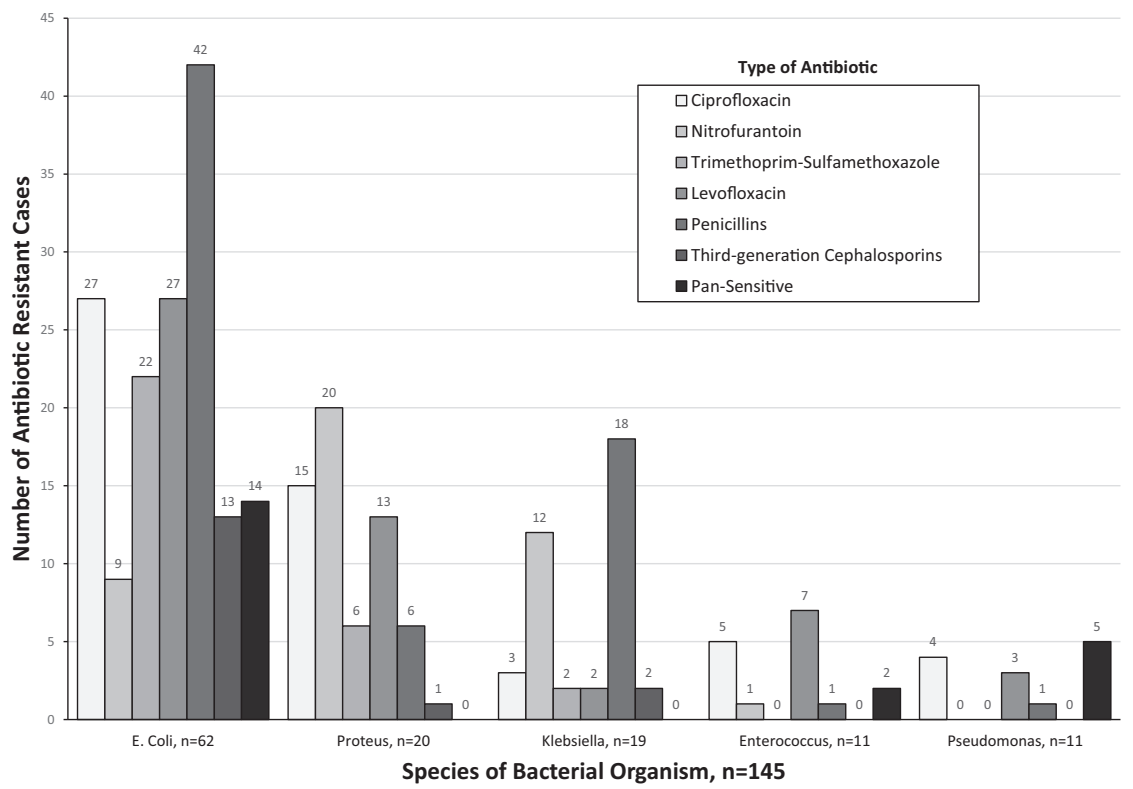

Figure 1. Frequency of resistant bacteria by type of antibiotic across positive* urine cultures $(\mathrm{n}=145)^{\dagger}$. *Positive Cultures defined as $\geq 100,000 \mathrm{CFU} / \mathrm{mL}$ for clean-catch specimens and $\geq 10,000 \mathrm{CFU} / \mathrm{mL}$ for catheterized specimens. ${ }^{\dagger}$ Each bar in this graph represents the number of cases when a positive culture was resistant to that type of antibiotic across the five most common types of bacterial organisms.

Administration released an updated warning around fluoroquinolones, stating the serious side effects seen outweigh the benefits of use. ${ }^{43}$ In addition, providers in our study prescribed overly long courses of antibiotics, over a quarter prescribed for more than 1 week, although guidelines recommend durations longer than 7 days in only a minority of cases. ${ }^{24,44-46}$ One explanation for the longer duration of treatment may be that $\mathrm{NH}$ providers are concerned about complicated UTIs such as CAUTIs. The duration for antibiotic treatment for CAUTIs is somewhere between 5 and 14 days per various guidelines. ${ }^{47}$ We found the duration of antibiotics was a little over a day longer between those with and without catheters (both averaged over 7 days), suggesting that possible concerns over complicated infections were less likely. IDSA recommends the shortest effective duration of antibiotic treatment possible, ${ }^{48}$ noting that shorter duration does not increase treatment failure nor increase adverse clinical events in older women with UTIs. ${ }^{24}$

The generalizability of our findings is limited by geographic locale and number of NHs. The study NHs, however, did not differ significantly from all NHs nationally. ${ }^{49}$ In addition, because we relied on the infection log to identify eligible residents, we cannot comment on any cases or residents that failed to make the infection log. Also, for a few variables, we had more than $10 \%$ missing data, rendering uncertain the true frequency of some findings, such as prescriber type. Certain data elements for sepsis, such as heart rate and respiratory rate were not included in the chart abstraction, making it difficult to determine UTI versus more complicated infections such as pyelonephritis, which might argue for a longer duration of antibiotic treatment. Lastly, we did not look for resolution, continuation, or development of symptoms after the antibiotic.

\section{CONCLUSION}

This study revealed that non-specific signs and symptoms were most often found in cases of presumed UTIs; that $\mathrm{NH}$ providers represented the largest group of antibiotic prescribers; that prescribers often failed to discontinue antibiotics even when clinically reasonable; and that nonrecommended antibiotics were often prescribed and for overly long durations. Three ways to improve guideline adherence are: (1) providing educational activities that specifically target $\mathrm{NH}$ nurses and $\mathrm{NH}$ providers to reduce the over-reliance on non-specific signs/symptoms and urine culture results, (2) encouraging antibiotic cessation in the face of negative cultures, and (3) improving the antibiotic choice and shortening the antibiotic duration. Implementing antibiotic stewardship programs offers an evidencebased way to increase the appropriate use of antibiotics. ${ }^{50}$

\section{ACKNOWLEDGMENTS}

Author Contributions: Conceptualization/design: CK, SZ, DW, PS, MM; Obtaining Funding: SZ and PS; Data collection and management: CK, KS, KW, PS; Guidance/interpretation of data analysis: CK, SZ, KS, KW, DW, DR, MM, PS; Statistical analysis: DR and CK; Manuscript writing: CK, SZ, KS, PS; Approval of final manuscript: CK, SZ, KS, KW, DW, DR, MM, PS.

Funding: Financial support for this study was provided entirely by a grant from the United States Agency for Healthcare Research and Quality (\#R18 HS022846-01). The funding agreement ensured the authors' independence in designing the study, interpreting the data, writing, and publishing the report. All authors report no conflict of interest. 


\section{Sponsor's Role: All authors report no conflict of interest.}

\section{REFERENCES}

1. Foxman B. The epidemiology of urinary tract infection. Nat Rev Urol 2010;7:653-660.

2. Litwin MS, Saigal CS, Yano EM et al. Urologic diseases in America Project: Analytical methods and principal findings. J Urol 2005;173:933-937.

3. Woodford HJ, George J. Diagnosis and management of urinary infections in older people. Clin Med (Lond) 2011;11:80-83.

4. Nace DA, Drinka PJ, Crnich CJ. Clinical uncertainties in the approach to long term care residents with possible urinary tract infection. J Am Med Dir Assoc 2014;15:133-139.

5. Rowe TA, Juthani-Mehta M. Diagnosis and management of urinary tract infection in older adults. Infect Dis Clin North Am 2014;28:75-89.

6. Phillips CD, Adepoju O, Stone N et al. Asymptomatic bacteriuria, antibiotic use, and suspected urinary tract infections in four nursing homes. BMC Geriatr 2012;12:1-8.

7. Nicolle LE, Zhanel GG, Harding GK. Microbiological outcomes in women with diabetes and untreated asymptomatic bacteriuria. World J Urol 2006;24:61-65.

8. Midthun S, Paur R, Bruce AW et al. Urinary tract infections in the elderly: A survey of physicians and nurses. Geriatr Nurs 2005;26:245-251.

9. Ninan S, Walton C, Barlow G. Investigation of suspected urinary tract infection in older people. BMJ 2014;349:g4070.

10. D'Agata E, Loeb MB, Mitchell SL. Challenges in assessing nursing home residents with advanced dementia for suspected urinary tract infections. J Am Geriatr Soc 2013;61:62-66.

11. Longo DR, Young J, Mehr D et al. Barriers to timely care of acute infections in nursing homes: A preliminary qualitative study. J Am Med Dir Assoc 2002;3:360-365.

12. Fleming A, Bradley C, Cullinan S et al. Antibiotic prescribing in long-term care facilities: A meta-synthesis of qualitative research. Drugs Aging 2015;32:295-303.

13. Levy CR, Eilertsen T, Kramer AM et al. Which clinical indicators and resident characteristics are associated with health care practitioner nursing home visits or Hospital transfer for urinary tract infections? J Am Med Dir Assoc 2006;7:493-498.

14. Loeb M, Brazil K, Lohfeld L et al. Effect of a multifaceted intervention on number of antimicrobial prescriptions for suspected urinary tract infections in residents of nursing homes: Cluster randomised controlled trial. BMJ 2005;331:669.

15. Boscia JA, Kobasa WD, Abrutyn E et al. Lack of association between bacteriuria and symptoms in the elderly. Am J Med 1986;81:979-982.

16. Walker S, McGeer A, Simor AE et al. Why are antibiotics prescribed for asymptomatic bacteriuria in institutionalized elderly people? A qualitative study of physicians' and nurses' perceptions. CMAJ 2000;163:273-277.

17. Kistler CE, Sloane PD, Platts-Mills TF et al. Challenges of antibiotic prescribing for assisted living residents: Perspectives of providers, staff, residents, and family members. J Am Geriatr Soc 2013;61:565-570.

18. Juthani-Mehta M, Drickamer MA, Towle V et al. Nursing home practitioner survey of diagnostic criteria for urinary tract infections. J Am Geriatr Soc 2005;53:1986-1990.

19. Croskerry P. Clinical cognition and diagnostic error: Applications of a dual process model of reasoning. Adv Health Sci Educ Theory Pract 2009;14 (Suppl 1):27-35

20. Juthani-Mehta M, Tinetti M, Perrelli E et al. Diagnostic accuracy of criteria for urinary tract infection in a cohort of nursing home residents. J Am Geriatr Soc 2007;55:1072-1077.

21. Loeb M, Bentley DW, Bradley S et al. Development of minimum criteria for the initiation of antibiotics in residents of long-term-care facilities: Results of a consensus conference. Infect Control Hosp Epidemiol 2001;22:120-124.

22. Sievert DM, Ricks P, Edwards JR et al. Antimicrobial-resistant pathogens associated with healthcare-associated infections summary of data reported to the National Healthcare Safety Network at the Centers for Disease Control and Prevention, 2009-2010. Infect Control Hosp Epidemiol 2013;34:1-14.

23. Sloane PD, Kistler C, Mitchell CM et al. Role of body temperature in diagnosing bacterial infection in nursing home residents. J Am Geriatr Soc 2014;62:135-140

24. Vogel T, Verreault R, Gourdeau M et al. Optimal duration of antibiotic therapy for uncomplicated urinary tract infection in older women: A double-blind randomized controlled trial. CMAJ 2004;170:469-473.
25. Nicolle LE. Urinary tract infections in the elderly. Clin Geriatr Med 2009;25:423-436.

26. Filice GA, Drekonja DM, Thurn JR et al. Diagnostic errors that lead to inappropriate antimicrobial use. Infect Control Hosp Epidemiol 2015;36:949-956

27. Olsho LE, Bertrand RM, Edwards AS et al. Does adherence to the Loeb minimum criteria reduce antibiotic prescribing rates in nursing homes? J Am Med Dir Assoc 2013;14(309):e301-e307.

28. Lim VH, Whitehurst T, Usoro E et al. Management of urinary tract infections in elderly patients: Strategies for improvement. BMJ Qual Improv Rep 2014;3:u203314.w201503.

29. Sundvall PD, Ulleryd P, Gunnarsson RK. Urine culture doubtful in determining etiology of diffuse symptoms among elderly individuals: A crosssectional study of 32 nursing homes. BMC Fam Pract 2011;12:1-7.

30. Jump RL, Crnich CJ, Nace DA. Cloudy, foul-smelling urine not a criteria for diagnosis of urinary tract infection in older adults. J Am Med Dir Assoc 2016;17:754.

31. Marasinghe KM. Computerised clinical decision support systems to improve medication safety in long-term care homes: A systematic review. BMJ Open 2015;5:e006539.

32. Field TS, Rochon P, Lee M et al. Computerized clinical decision support during medication ordering for long-term care residents with renal insufficiency. J Am Med Inform Assoc 2009;16:480-485.

33. Meeker D, Linder JA, Fox CR et al. Effect of behavioral interventions on inappropriate antibiotic prescribing among primary care practices: A randomized clinical trial. JAMA 2016;315:562-570.

34. McDonagh M, Peterson K, Winthrop $\mathrm{K}$ et al. Improving Antibiotic Prescribing for Uncomplicated Acute Respiratory Tract Infections, Vol 15, Comparative Effectiveness Review No. 163. Rockville, MD: Agency for Healthcare Research and Quality, 2016. Available from: https://www.ncbi. nlm.nih.gov/books/NBK344270/

35. Leis JA, Rebick GW, Daneman N et al. Reducing antimicrobial therapy for asymptomatic bacteriuria among noncatheterized inpatients: A proof-ofconcept study. Clin Infect Dis 2014;2014(58):980-983.

36. Gilbert GL. Knowing when to stop antibiotic therapy. Med J Aust 2015;202:121-11

37. Zarowitz BJ, Allen C, Tangalos $\mathrm{E}$ et al. Algorithms promoting antimicrobial stewardship in long-term care. J Am Med Dir Assoc 2016;17:173-178.

38. Fakih MG, Rey JE, Pena ME et al. Sustained reductions in urinary catheter use over 5 years: Bedside nurses view themselves responsible for evaluation of catheter necessity. Am J Infect Control 2013;41:236-239.

39. Prevention CfDCa. The Core Elements of Antibiotic Stewardship in Nursing Homes. Atlanta, GA: US Department of Health and Human Services, CDC; 2015. Available at: http://www.cdc.gov/longtermcare/index.html

40. American Thoracic Society; Infectious Diseases Society of America. Guidelines for the management of adults with hospital-acquired, ventilator-associated, and healthcare-associated pneumonia. Am J Respir Crit Care Med 2005;171:388-416.

41. Gupta K, Hooton TM, Naber KG et al. International Clinical Practice Guidelines for the treatment of acute uncomplicated cystitis and pyelonephritis in women: A 2010 update by the Infectious Diseases Society of America and the European Society for Microbiology and Infectious Diseases. Clin Infect Dis 2011;52:e103-e120.

42. Iannini PB. The safety profile of moxifloxacin and other fluoroquinolones in special patient populations. Curr Med Res Opin 2007;23:1403-1413.

43. FDA updates warning for fluoroquinolone antibiotics. In: Administration USFaD, ed: FDA New Release, Silver Spring, MD, 2016.

44. Nicolle LE, Bentley DW, Garibaldi R et al. Antimicrobial use in long-termcare facilities. SHEA Long-Term-Care Committee. Infect Control Hosp Epidemiol 2000;21:537-545.

45. Nicolle LE, Louie TJ, Dubois J et al. Treatment of complicated urinary tract infections with lomefloxacin compared with that with trimethoprimsulfamethoxazole. Antimicrob Agents Chemother 1994;38:1368-1373.

46. Lutters M, Vogt-Ferrier NB. Antibiotic duration for treating uncomplicated, symptomatic lower urinary tract infections in elderly women. Cochrane Database Syst Rev 2008:CD001535.

47. Trautner BW. Management of catheter-associated urinary tract infection. Curr Opin Infect Dis 2010;23:76-82.

48. Barlam TF, Cosgrove SE, Abbo LM et al. Implementing an antibiotic stewardship program: Guidelines by the Infectious Diseases Society of America and the Society for Healthcare Epidemiology of America. Clin Infect Dis 2016;62:e51-e77.

49. Nursing Home Compare. 2015. Available at https://www.medicare.gov/ nursinghomecompare/search.html. Accessed August 15, 2015.

50. Sloane PD, Huslage K, Kistler CE et al. Optimizing antibiotic use in nursing homes through antibiotic stewardship. N C Med J 2016;77:324-329. 